\title{
EXPRESSIVE LIGHTING FOR INTERACTIVE ENTERTAINMENT
}

\author{
Magy Seif El-Nasr \\ Northwestern University \\ magy@northwestern.edu
}

\author{
Ian Horswill \\ Northwestern University \\ ian@cs.northwestern.edu
}

\begin{abstract}
Lighting design is an essential element of visual storytelling. In cinematic theory, lighting design plays an important role in illumination, directing viewer's gaze to important locations, and portraying visual tension. However, lighting design is a complex process, and is especially problematic for interactive entertainment systems, since important design parameters such as spatial configuration, dramatic focus, and dramatic intensity, cannot be determined in advance. Indeed, they often change dramatically during interaction. Additionally, manually adjusting colors, positions, and angles of each light in a scene is a time consuming and tedious process. In this paper, we describe a system developed based on cinematic and theatrical lighting design theory to automatically adjust positions, colors, and angles of each light in real-time to accommodate the continually evolving dramatic situation, while maintaining the desired style, and ensuring visual continuity.
\end{abstract}

\section{INTRODUCTION}

Visual presentation is a very important component of visual storytelling. Lighting, in particular, is important, because it achieves many artistic goals, such as providing mood, emphasizing dramatic tension, and directing the viewer's attention to the current dramatic focus (i.e. important characters or objects) [1-3]. Lighting designers vary angle, placement, and color of each light in the scene to achieve these goals. For example, they vary light angles relative to characters' faces to evoke mystery and uneasiness [4].

Creating a lighting design that achieves these artistic goals in an interactive scene is difficult because important design elements, such as character positions and the dramatic significance of an event, are not known at design time. As a consequence, many game designers use static lighting where light placement is based on set design (level design) [5,6]. This prevents the use of light to communicate tension or action. Worse yet, camera and character movement may render some characters intermittently invisible.

This paper presents a system that adapts cinematic and theatrical lighting design theory $[9,10]$ to interactive settings. We begin by formalizing the rules of lighting design theory as a set of cost functions. We then present a system that uses non-linear optimization to select light angles and colors in real-time to suit the evolving dramatic situation while maintaining a lighting style, ensuring visual continuity, and accommodating movement and action.

\section{RELATED WORK}

Most game designers use static lighting, such as lighting based on level design or ambient lighting. Ambient lighting is simple and provides good visibility. However, it has no cinematic quality and contributes little to the overall aesthetic and dramatic qualities of the piece [7]. It is, however, well suited for toy-like interactive environments, such as the Sims. In games, such as Max Payne, Quake, Half-life, and Devil May Cry, designers manually place lights and set their orientations and colors $[5,6]$. While this scheme provides a workable solution for most games, it is inflexible. First, it doesn't show the depth or the three dimensional nature of the character. Second, it does not parallel the dramatic structure of the interaction since colors and angles are not dynamically adjusted during interaction. Also, in some situations the character may not be sufficiently lit; a problem that surfaces in many games, e.g. The Thing.

Tomlinson [8] developed a system that selects camera movements and light colors to portray the emotions of characters residing in an interactive world. For example, the system uses low camera angle to show that a character is powerful, or harsh red light to make a character look demonic. He divided lights into two categories: global and personal lights. Global lights are static and are used to provide the main source of illumination. Personal lights are fixed on characters; their colors are computed depending on the emotional state of the character. This design provides only a visual message of the characters' emotional state. Cinematic goals of lighting design, such as portraying dramatic intensity or emphasizing dramatic focus, were not addressed. Alternatively, we propose a system that extends the purpose of lighting to incorporate lighting design goals as addressed by lighting designers in theatre, film, and computer animation [1-3].

\section{EXPRESSIVE LIGHTING}

B. Foss [11] defined several goals for narrative events and their filmic presentation. We extend these goals to define expressive lighting. Expressive lighting achieves realistic, lyrical, dramatic, and aesthetic goals. A lighting design achieves realistic goals when it conforms to a realistic color palette, and when selected angles adhere to the direction of established practical ${ }^{1}$ sources. A lighting design achieves lyrical goals when designed to evoke moods or emotions from the audience. Lighting designers use a variety of perceptual rules to adjust the colors and angles of lights to achieve the desired mood [12, 13].

A lighting decision achieves a dramatic goal when it influences characters' relations and choices, and when it emphasizes dramatic tension and action. In interactive scenes, lighting, among other visual elements, can be used to influence participants' choices and actions [6]. Many game designers vary light contrast and colors to direct the participant's attention to important artifacts. Such lighting effects, among others, may indirectly affect participants' choices and actions. A lighting configuration can emphasize a dramatic tension by providing color contrast that parallels the scene's dramatic intensity.

\footnotetext{
${ }^{1}$ local sources that exist in the scene, e.g. torches and windows.
} 
Lighting designers also consider several aesthetic qualities when configuring lights for a scene. These qualities include modeling, depth, and style. Depth is often established by selecting different brightness values for the colors of lights lighting the background and those lighting the foreground.

Lighting designers change light colors and angles unobtrusively and carefully so that the change wouldn't break the suspension of disbelief. This, however, does not entail that radical changes are never used. In fact, the level of change allowed depends on the camera movement and style. For example, when using an expressionistic style, it is acceptable that a designer changes background colors from an extreme warm to an extreme cool color between shots. Such changes are noticeable, but they often don't break the suspension of disbelief, because they are consistent with the style used in the scene. Such techniques are evident in several movies, such as Moulin Rouge.

\section{ELE -EXPRESSIVE LIGHTING ENGINE}

ELE is designed to select a lighting configuration, colors, and angles in real-time. ELE is given some parameters: a set of parameters describing style, list of practical sources in the scene, dimensions of the stage and the characters in the scene, and the dramatic intensity of the situation. Using these parameters ELE computes the number of lights used. For each of these lights, it computes the type of instrument (e.g. spot light or point light), color in RGB color space, attenuation, position, orientation, range, masking parameters, and, depending on the light instrument used, penumbra and umbra angles. These parameters are then given to a rendering engine to render the frame.

ELE is composed of three subsystems: a system that determines the number of lights used and the areas they affect, a color optimization system that selects colors for each light, and an angle optimization system that selects angles for each light. The system interacts with the camera to request camera cuts when necessary changes are distracting.

The lighting system divides the visible area into $n$ different areas. It categorizes them as: focus (the focus of the scene), non-focus (areas surrounding the focus area), and background areas. It assigns lights to each area depending on its category. The angle and color systems then assign angles and colors for each light.

In the following sections, we will describe the angle and color systems since these portray the most visible changes in the scene. Other aspects of the system, such as configuring lights and controlling the camera and character behaviors, are left out due to restrictions of space. However, interested readers are referred to [14] for a more detailed description of these systems.

\subsection{Angle optimization system}

The system selects an angle for each $k^{2} y^{2}$ light according to several requirements, which include ensuring visual continuity, maintaining the illusion of a practical source, providing mood, and ensuring that all characters are visible.

Cinematic rules used to satisfy these requirements often contradict with one another. Angles used to establish mood, for example, don't usually produce good visibility, e.g. rim or silhouette angles. Thus, we softened these rules into cost functions, where the designer controls weights associated with

\footnotetext{
${ }^{2}$ the main source of light that establishes direction and shadows
}

the contradicting requirements, such as mood, visibility, and modeling. ELE then uses optimization to find the best solution (i.e. solution with minimum cost).

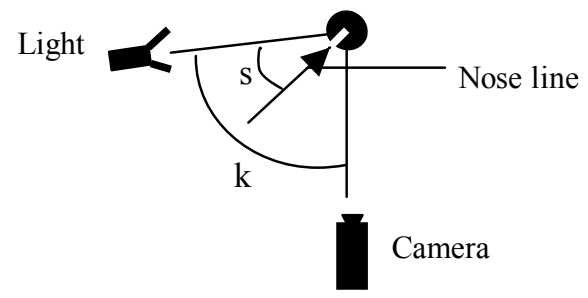

Figure 1. Angles between subject, camera, and light

ELE uses the following parameters:

- Cost associated with deviation from angle used in previous frame (how much artists care about visual continuity)

- Cost associated with motivation (describes how much artists care about simulating effects of practical sources)

- Cost of visibility (how much artists care about visibility)

- Cost associated with mood (how much artists care about mood)

- Ideal mood angle (the ideal mood angle; e.g. side-light)

These parameters are used as weights in the following cost function:

$$
\begin{gathered}
\operatorname{cost}\left(k, s, k^{-}, m\right)=\lambda_{v}(1-V(k, s))+\lambda_{-}\left|k-k^{-}\right|+ \\
\lambda_{m}|k-m|+\lambda_{l} \min _{i}\left|k-l_{i}\right|,
\end{gathered}
$$

where $k$ and $s$ are defined as the key light azimuth angle relative to the camera and the subject angle relative to the key light, respectively, as shown in figure $1, k^{-}$is the key light azimuth angle used in the previous frame, $\lambda_{-}$is the cost of changing the key light angle over time (to enforce visual continuity), $\lambda_{m}$ is the cost of deviation from the mood azimuth angle, $m$ is the mood azimuth angle suggested by the artist, $\lambda_{l}$ is the cost of azimuth angle deviation from a practical source direction, $l_{i}$ is the azimuth angle of light emitted by the practical source $i$, and $\lambda_{v}$ is the cost of deviation from an orientation of light that establishes best visibility and modeling.

Based on Millerson's documented rules [9], we formulated the following equation to evaluate the visibility and modeling of a given key light azimuth angle:

$$
V(k, s)=\sin (k) \cos (s) \text {. }
$$

Millerson recommended an elevation angle between $\pi / 6$ and $\pi / 3$.

ELE uses a non-linear optimization system based on hill climbing to select an angle for each key light that minimizes the cost function above.

ELE uses rules based on Millerson's [9] guidelines to select fill and backlight azimuth angles depending on the value of the key light angle. According to Millerson's guidelines [9], fill light azimuth and elevation angles are calculated to be the mirror image of the key light angle. We define backlight azimuth angle as:

$$
b=(k+\pi) \bmod 2 \pi .
$$




\subsection{Color optimization system}

The interaction between colors assigned for each area in a scene composes the contrast and feeling of the entire image. Thus, we differentiate between the three types of areas: background, focus, and non-focus. We then calculate contrast and depth as the difference between colors assigned to each of these areas. We also allow the designers to specify several costs and ideal color values for each area category as follows:

- Color ideal values: Hue, Saturation, Warmth, and Lightness for each area category (focus, non-focus, background), and depth

- Costs of deviation from the specified color ideal values

- Color limits, restricting a color palette

- Depth ideal value

- Depth cost (how much the artist cares about depth)

- Visual continuity cost (how much the artist cares about visual continuity)

- Contrast style (i.e. saturation contrast, lightness contrast, or warmth/coolness contrast)

Using the ideal values and their associated costs, the system uses constrained non-linear optimization to select a color for each individual light in the scene that will minimize the cost function:

$$
\begin{aligned}
& \operatorname{cost}\left(c^{t}, c^{t-1}\right)= \\
& P\left(c^{t}\right)+\left(\sum_{i \in\{f, n, b\}}\left(\lambda_{c h} E\left(c_{i}^{t}, c_{i}^{t-1}\right)+Q\left(c_{i}^{t}\right)\right)\right)+\varepsilon \sum_{j} \log \left(-g_{j}(x)\right),
\end{aligned}
$$

where $g: \mathrm{R}^{3} \rightarrow[0,1]$ describes the constraints on the colors used. P and $\mathrm{Q}$ are defined as follows:

$$
\begin{gathered}
P\left(c^{t}\right)=\lambda_{d}\left(D\left(c^{t}\right)-d\right)^{2}+\lambda_{c}\left(\operatorname{contrast}_{\phi}\left(c^{t}\right)-\delta\right)^{2}, \\
Q\left(c_{i}^{t}\right)=\begin{array}{l}
\lambda_{s_{i}}\left(S\left(c_{i}^{t}\right)-s_{i}\right)^{2}+\lambda_{h_{i}}\left(H\left(c_{i}^{t}\right)-h_{i}\right)^{2}+ \\
\lambda_{l_{i}}\left(L\left(c_{i}^{t}\right)-l_{i}\right)^{2}+\lambda_{w_{i}}\left(W\left(c_{i}^{t}\right)-w_{i}\right)^{2},
\end{array}
\end{gathered}
$$

where $S, H, L$, and $W$ are functions calculating saturation, hue, lightness, and warmth of a color using formulae defined in [15]. Contrast $_{\phi}$ defines color contrast as the difference in lightness, saturation, or warmth (depending on the contrast style selected) between colors of lights lighting the focus, $f$, non-focus, $n$, and background, $b$, areas. The color $c$ is a vector of colors of lights lighting $f, n$, and $b$. Subsequently, $h, s, l, w$, and their associated costs are specified for each area $f, n$, and $b$.

$E\left(c_{i}, c_{i-1}\right)$ denotes the color difference between color $c$ at frame $t$ and the previous frame, $t-1$. It is computed using CIEDE2000 formulated by CIE Lab $[17,18]$.

$D\left(c^{t}\right)$ measures depth as follows:

$$
D\left(c^{t}\right)=\sum_{i \in \text { background }} \sum_{j \notin \text { background }} E\left(c_{i}^{t}, c_{j}^{t}\right),
$$

We used linear regression to devise a formula that evaluates the warmth of a color in RGB color space; the formula is based on results collected by Katra and Wooten [16]:

$$
W\left(c^{t}\right)=\left[\begin{array}{c}
.0088 \\
.0006 \\
-.0105
\end{array}\right]\left[\begin{array}{l}
R \\
G \\
B
\end{array}\right]-0.422
$$

ELE uses gradient descent to search through a nine-dimension space of RGB values differentiating between colors of focus, nonfocus, and background areas. It selects RGB values for focus, non-focus, and background areas that minimize the cost function described above. In this case, local minima will suffice since we want to ensure visual continuity.

\subsection{Automatic adjustment of the design parameters}

As mentioned above, artists can control several parameters specifying the importance of the lighting design goals, as well as constraining the colors and the contrast. Manipulating these parameters for a complete scene is a very tedious and time consuming task. Therefore, ELE automatically selects values for these parameters depending on the current situation, current dramatic intensity, and the established style. It varies these parameters to accommodate the dramatic situation while also maintaining visual continuity. ELE uses a rule-based system, where rules are defined based on several documented cinematic conventions [9]. To retain artistic control when needed, ELE allows artists to override its decisions by authoring rules that trigger specific settings for the parameters.
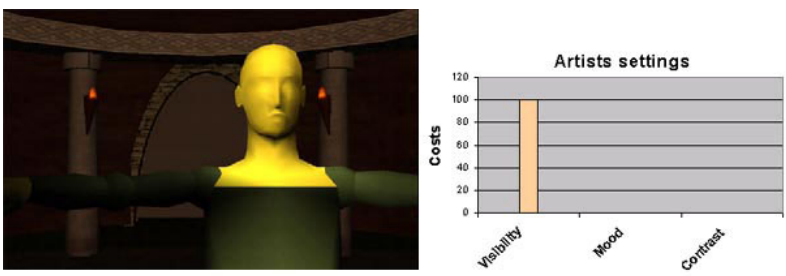

(a) Emphasis on visibility
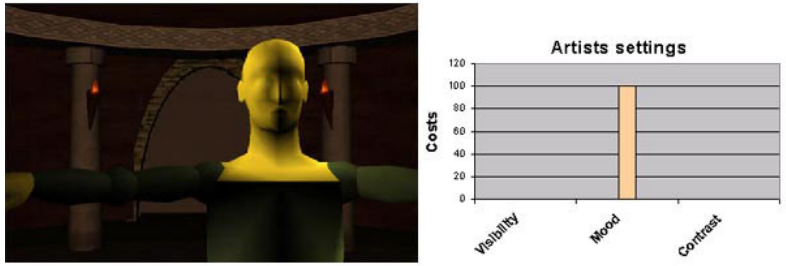

(b) Emphasis on mood, with a mood angle = side light
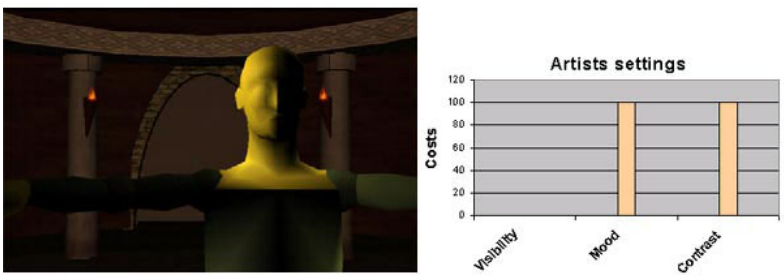

(c) Emphasis on mood, with high contrast, with a mood angle = side light
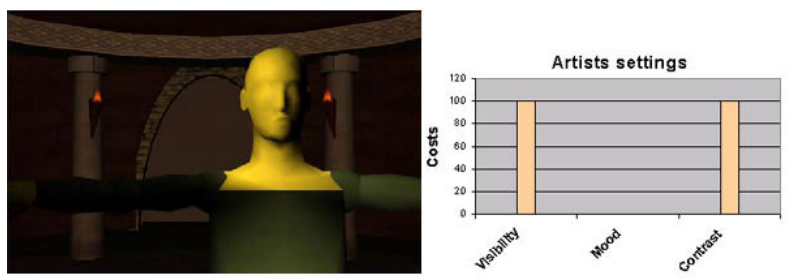

(d) Emphasis on visibility, with high contrast

Figure 2. Results of the angle optimization system (Contrast is calculated as the lightness difference between key and fill lights) 


\section{RESULTS}

ELE has been implemented. Figures 2 and 3 show few results of the angle and color systems. These figures illustrate the expressiveness and flexibility of the parameters engineered. The images in figure 2 show different light angles selected by ELE depending on some artistic settings specifying costs associated with visibility, mood, and contrast. Similarly, figure 3 shows the results of ELE's real-time adjustment of colors to accommodate artist's parameters concerning contrast, lightness, and warmth of areas around the character. These images show numerous effects that can be created using few artistic parameters.

The lighting system has also been implemented and tested within a complete scene from Mirage, an interactive story based on the Greek tragedy Electra. Interested readers are referred to [19] for a full video of the scene with different lighting styles.
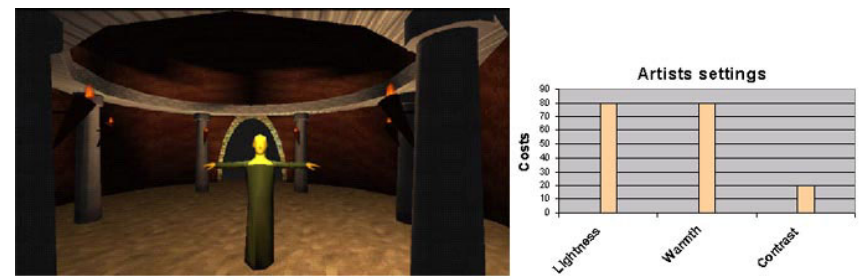

(a) Warm tones, low color contrast

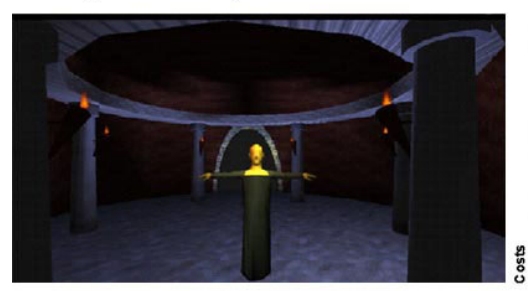

(b) Cool tones, high color contrast
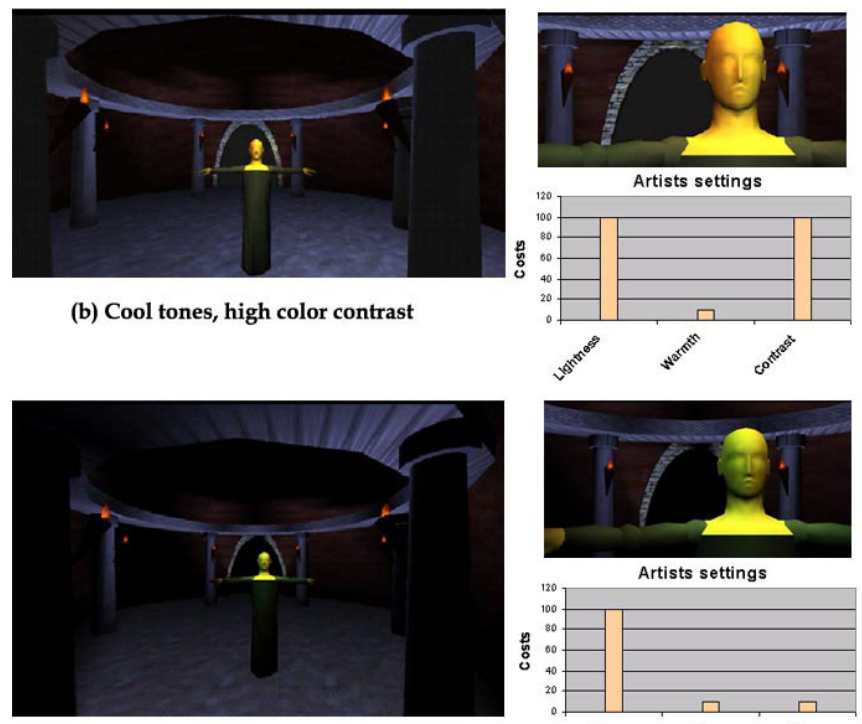

Artists settings

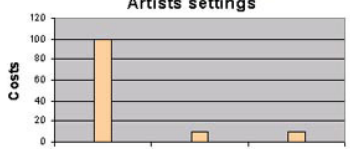

(c) Cool tones, low color contrast

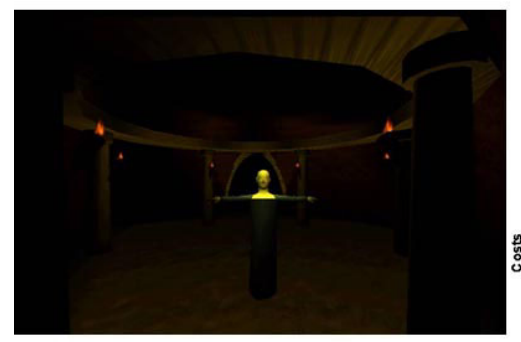

(d) Warm tones, high brightness contrast

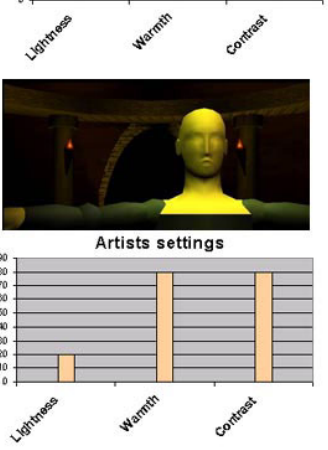

Figure 3. Results of the color optimization system (Contrast is calculated as the color difference between lights on the subject and other lights; lightness and warmth are costs associated with non-focus and background areas)

\section{CONCLUSION AND FUTURE WORK}

In this paper, we presented ELE - a lighting system based on rules and cost functions formulated using theatrical and cinematic lighting design theory. ELE is a tool that can be used to automate part of the tedious process of building an interactive narrative. It frees artists to concentrate on other aspects of the design such as interaction and character development. However, lighting is only one element of visual design. Visual storytelling involves several elements all working together to compose a scene. In the future, we would like to explore the role of camera movement and character blocking in interactive narrative.

\section{REFERENCES}

[1] S. Calahan, "Storytelling through lighting: a computer graphics perspective," presented at Siggraph Course Notes, 1996.

[2] B. Block, The Visual Story: Seeing the Structure of Film, TV, and New Media. New York: Focal Press, 2001.

[3] P. Ward, Picture Composition for Film and Television. Oxford: Focal Press, 1996.

[4] J. M. Gillette, Designing with Light, 3rd. ed. Mountain View, CA: Mayfield, 1998.

[5] A. Maattaa, "GDC 2002:Realistic Level Design for Max Payne," in Gamasutra, 2002.

[6] D. Carson, "Environmental Storytelling: Creating Immersive 3D Worlds Using Lessons Learned from the Theme Park Industry," in Gamasutra, 2000.

[7] J. Birn, "Digital Lighting \& Rendering," G. Maestri, Ed. Indianapolis: New Riders, 2000.

[8] W. M. Tomlinson, "Interactivity and Emotion through Cinematography." MIT Media Lab: MIT, 1999.

[9] G. Millerson, The Technique of Lighting for Telivision and Film, 3rd ed. Oxford: Focus Press, 1991.

[10] B. Brown, Motion Picture and Video Lighting. Boston: Focal Press, 1996.

[11] B. Foss, Filmmaking: Narrative \& Structural Techniques. Los Angeles: Silman-James Press, 1992.

[12] J. Alton, Painting with Light. Berkeley: University of California Press, 1995.

[13] D. Campbell, Technical Theatre for Non-technical People: Allworth Press, 1999.

[14] M. Seif El-Nasr, "ELE- Expressive Lighting Engine for Interactive Narrative," in Computer Science. Evanston, IL: Northwestern University, Forthcoming.

[15] K. R. Castleman, Digital Image Processing. Englewood Cliffs: Prentice Hall, 1996.

[16] E. a. W. Katra, B. R., "Preceived lightness/darkness and warmth/coolness in chromatic experience," 1995.

[17] B. H. Hill, Roger, T. , and Vorhagen, F. W., "Comparative Analysis of the Quantization of Color Spaces on the Basis of the CIELAB Color-Difference Formula," ACM Transactions on Graphics, vol. 16, pp. 109-154, 1997.

[18] M. R. Luo, Cul, G., and Rigg, B., "The Development of CIE 2000 Colour Difference Formula: CIEDE2000," vol. 2000: CIELAB, 2000.

[19] M. Seif El-Nasr, "www.cs.northwestern.edu/ magy/ ele.html," Seif El-Nasr, Magy,

2002. 